\title{
Aperçu sur la composition, l'origine et la formation de la faune cavernicole de la Stara planina occidentale (Bulgarie)
}

\author{
par \\ Petar BERON*
}

SUMMARY

\section{On the Composition, the Origin and the Formation of the Cave Fauna of Western Stara Planina (Bulgaria)}

The karstic area between the rivers Timok and Vit has about 500 caves and pot-holes, more than 300 of them being the subject of the present study. In this area (called here Western Stara Planina) we have noted 63 species and subspecies of terrestrial troglobites and 17 species and subspecies of aquatic troglobites. The two Regions into which we split the area (region of Ogosta and Region of Iskar) are the richest in Bulgaria in cave fauna.

This study deals with the particularities in the composition and the distribution of the different groups of terrestrial troglobites, and especially of such groups as Diplopoda, Isopoda, Coleoptera and others. According to the paleogeographic development of the area conclusions are made concerning the age of certain troglobites and the barriers determining their present distribution.

La Stara planina occidentale est la région la plus riche en grottes et gouffres parmi les régions kartiques de Bulgarie. On connaît dans ce territoire (entre les rivières Timok et Vit) plus de 500 grottes (s.lato) dont 350 sont plus ou moins prospectées au point de vue biospéléologique (y compris env. 300 par l'auteur lui-même). D'après le nombre de ses troglobies terrestres (63 espèces et sousespèces) et aquatiques (17 esp.et sous-esp.) la Stara planina occidentale est également la région la plus riche de Bulgarie.

Nos recherches dans cette région (avec le concours des collègues $P$. Tranteev, V. Beskov, V. Guéorguiev, Hr. Deltshev, St. Andreev, A. Popov, T. Micev, M. Kwartirnikov et autres) nous ont permis de découvrir tout un complexe d'espèces, dont plusieurs sont des nouveautés. Les résultats de la détermination du matériel récolté (dus à plusieurs éminents spécialistes) font partie du Catalogue de la faune cavernicole bulgare (Guéorguiev et Beron, 1962) et de ses suppléments (Beron et Guéorguiev, 1967, Beron, 1972). En nous appuyant sur les données obtenues, nous avons essayé de subdiviser le partie occidentale de la

*Institut de Zoologie, Boul. Rousski 1, 1000 Sofia, Bulgaria 


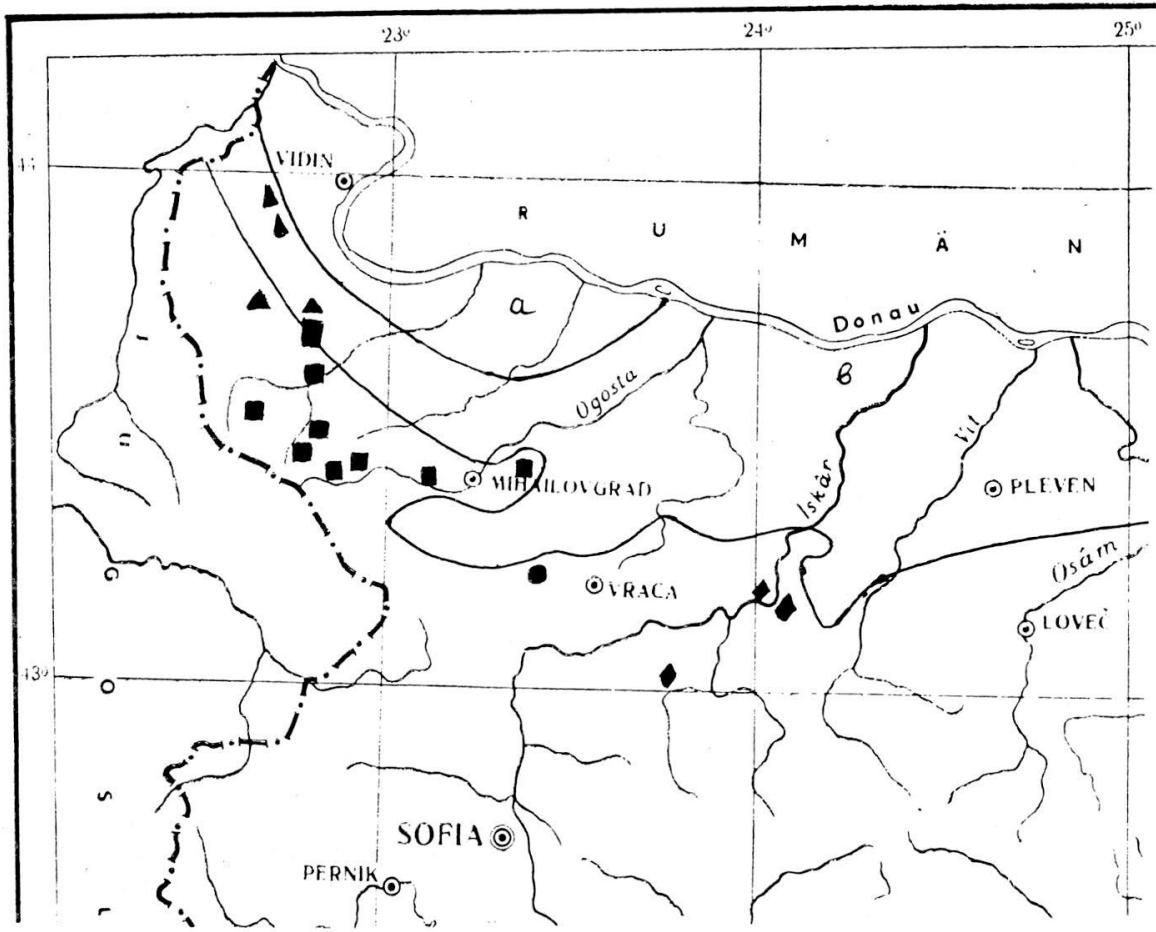

Fig. 1 - Bulgarie du Nord-Ouest pendant le Pontien - a

Bulgarie du Nord-Quest pendant le Tortonien - b

$\Delta$ - répartition de Hyloniscus flammula

- répartition de Cyphonisculleus gueorguievi

- répartition de $C$. bulgaricus

- répartition de Tricyphoniscus bureschi

Stara planina en régions biospéléologiques (Beron, 1976). Ici nous allons procéder à une revue plus détaillée des divers groupes de cavernicoles peuplant les grottes entre les rivières Timok et Vit.

Gastropoda. On ne connaît pas de troglobies parmi les Gastropodes terrestres peuplant les grottes du territoire étudié (indiqué plus loins comme "Bulgarie du Nord-Ouest"). L'espèce la plus répandue est l'Oxychilus glaber striatus.

Isopoda. Nous connaissons 8 troglobies du sous-ordre Oniscoidea dans ce territoire (sur 18 troglobies bulgares de ce groupe). Elles appartiennent toutes à la famille des Trichoniscidae (les genres Trichoniscus, Hyloniscus, Bureschia, Balkanoniscus, Tricyphoniscus, Cyphoniscellus) et ont des aires de répartition assez instructies.

Trichoniscus bononiensis habite pratiquement toutes les grottes près de Belogradcik et des villages Oresec, Repljana, Salas et autres. Elle fait défaut dans les grottes de Rabiska mogila et des endroits plus au Nord, ainsi que dans les 


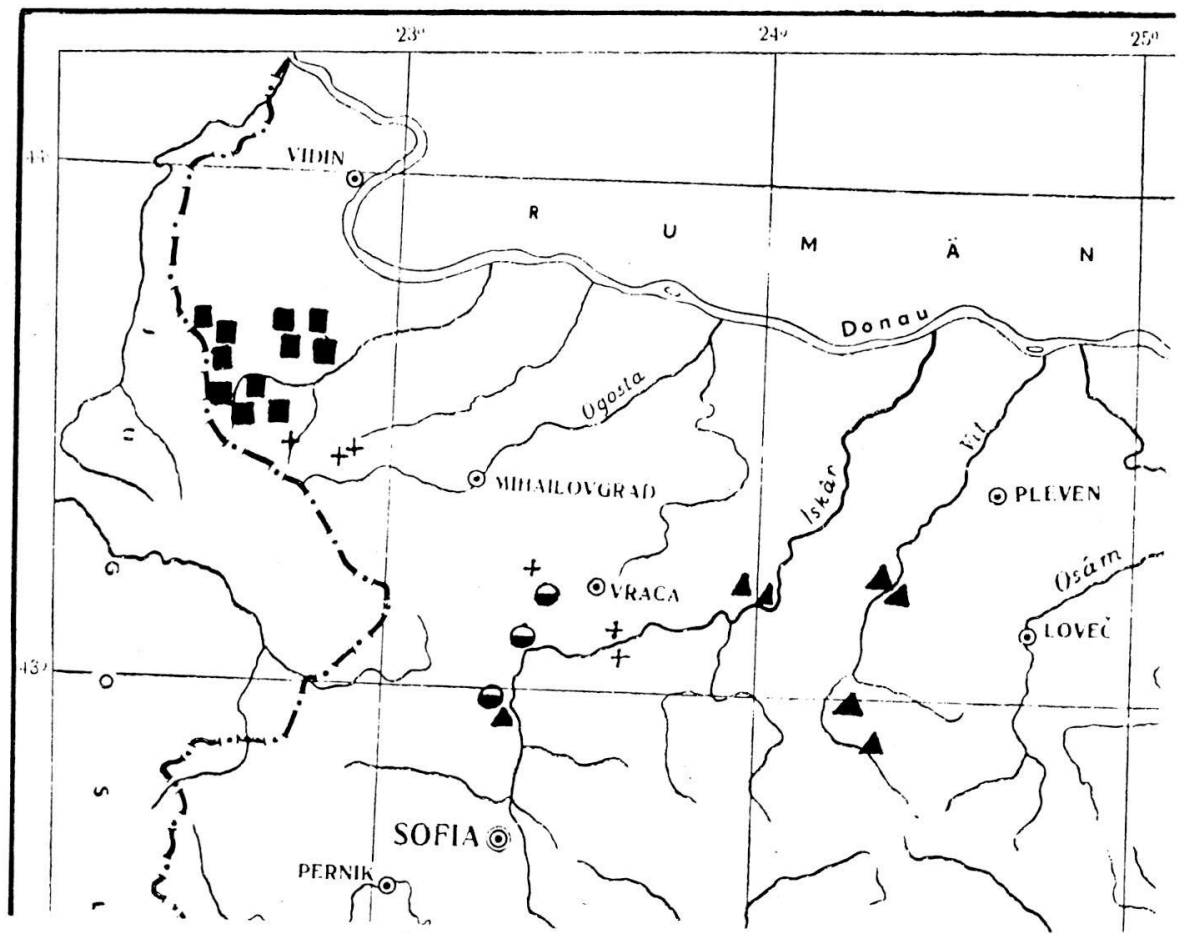

Fig. 2 - + - répartition de Trichoniscus anophthalmus

- répartition de Trichoniscus bononiensis

- répartition de Bureschia bulgarica

$\boldsymbol{\Delta}$ - répartition de Balkanoniscus corniculatus

multiples grottes au Sud-Est de Repljana, où $T$. anophthalmus se substitue à celle-ci. Selon Andreev (in litt.), T. bononiensis serait proche de T. bogovinae, décrite récemment par Pljakić de la Serbie orientale. Il est à noter que dans aucune grotte parmi le grand nombre de celles-ci, étudiées en Bulgarie du NordOuest, nous n'avons observé deux espèces de Trichoniscinae troglobies habitant ensemble, tandis que c'est presque une règle de trouver dans chaque grotte une espèce de Trichoniscinae et une espèce de Haplophthalminae. En allant du Nord vers le Sud les grottes sont peuplées par des Isopodes comme suit: Hyloniscus flammula, ensuite Trichoniscus bononiensis, suivie par T.anophthalmus. Par endroits on trouve aussi le Trichoniscus bureschi, considéré comme troglophile (toujours séparement d'autres Trichoniscinae.).

Parmi les Hyloniscus (genre comprenant une trentaine d'espéces) il n'y a que $H$. flammula et deux espèces japonaises qui soient aveugles (Vandel, 1968, 1969). $H$. flammula habite (en nombre considérable) les grottes de Rabiska mogila, la grotte Elenova dupka près du v. Bela, la grotte Studena près du v. Vojnica et la grotte Varkan près du v.Druzba. Ces trois dernières localités se trouvent sur 
les terrains submergés pendant la transgression maximale (tortonienne). Les villages Bela et Granitovo sont situés non loin l'un de l'autre, mais la grotte Cankinoto vrelo prés de Granitovo (vraisemblablement n'ayant pas été submergée au Néogène) est habitée par Trichoniscus bononiensis, tandis que Elenova dupka près de Bela abrite Hyloniscus flammula. On peut supposer que $H$.flammula est un troglobie plus récent que $T$. bononiensis, ayant habité (avant de pénétrer dans les grottes) les alentours de Rabisa. Ce n'est qu'après que la mer Tortonienne se fut rétirée qu'elle a pu effectuer une migration vers le Nord et envahir les grottes nouvellement formées dans le territoire exondé. Cette extension n'a pu avoir lieu qu'à partir du Pliocène supérieur, voire dans le Pleistocène, étant donné que les localités Studena et Varkan n'ont émergé qu'après le Pontien.

Nous avons trouvé dans la grotte Pesterata v Dedin dol près du v.Komstica aussi l'espèce Hyloniscus stankovici (dét. St. Andreev), décrite par Pljakic de la Serbie orientale, mais elle a un grand ocelle. Les espèces roumaines H.flammuloides et H.dacicus, décrites par Tabacaru (1973), sont également oculées. Etant donné que la péninsule des Balkans est considérée comme un centre important de la formation du genre Hyloniscus, c'est bien probable que la forme ancestrale de toutes ces espèces ait habité les montagnes serbes.

Trichoniscus anophthalmus, que Vandel (1965) a décrit des grottes près de Cerepis, se trouve par places dans le territoire étudié. En 1967 le Prof. Vandel a décrit une autre forme qu'il a attribué à cette espèce ( $T$. anopthalmus intermedius). Cette forme provient de la grotte Tabacka près de Roussé, soit à plus de $200 \mathrm{~km}$ à l'Est de Cerepis. Vandel la considère comme un chaînon de liaison entre T.a. anophthalmus et T. bureschi. Cela nous paraît peu probable. Premièrement, T.a. anophthalmus et T. bureschi habitent d'une manière sympatrique la Stara planina occidentale, alors que leur "chainon intermédiaire" a été trouvé dans une localité assez excentrique de l'aire des deux espèces. Deuxièmement, le Prof. Vandel a fait connaître que l'endopodite du pléopode I du mâle de T.a. intermedius "est plus court et plus trapu que celui de bureschi et celui d'anophthalmus". Un tel changement "cyclique" de la forme du pléopode est difficile, d'après nous, d'être compris en rangeant ces taxa dans une lignée phylétique "bureschi - a. anophthalmus - a. intermedius". Il est possible que le troglobie $T$. anophthalmus soit dérivé effectivement du troglophile T. bureschi, mais il convient mieux de considérer "T.a. intermedius" a priori comme une espèce à part.

D'un autre côté, il est possible que $T$. bureschi s'avère un jour synonyme de $T$. inferus des Carpates roumaines (dont on connaît aussi des exemplaires aveugles). T. bureschi a été trouvé dans plusieurs grottes de Stara planina, aussi bien que dans deux grottes du Rhodope, mais toujours oculé. Cette espèce n'est pas signalée en dehors des grottes.

Balkanoniscus corniculatus habite des grottes très humides, parfois même inondées, parsemées le long des rivières Iskâ̂ et Vit. C'est un des trois genres troglobiontes de la Stara planina, représenté aussi dans le R hodope (B. beroni).

Bureschia bulgarica méne une vie amphibie - elle se trouve en grande quan- 


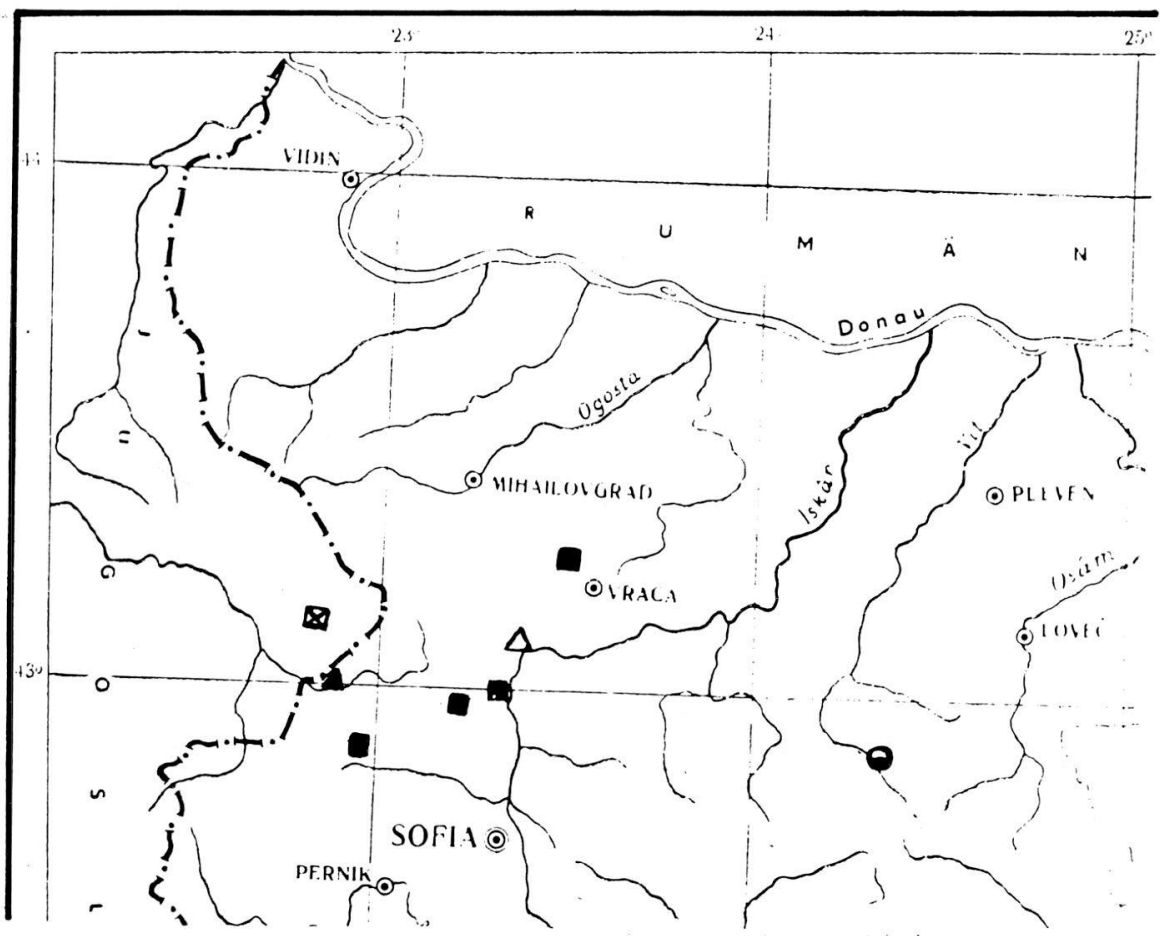

Fig. 3 - $\Delta$ - répartition de Stenasellus bureschi bureschi

$\triangle$ - répartition de Stenasellus bureschi lakatnicensis

- répartition de Sphaeromides bureschi

$\boldsymbol{\Delta}$ - répartition de Sphaeromides bureschi serbica

O - répartition de Sphaeromides polateni

tité près des lacs et des chutes d'eau dans quelques grottes sur la rive gauche de l'Iskar et dans la Vracanska planina. Elle rappelent les Titanethes et nous semblent être d'âge considérable.

Tricyphoniscus bureschi qui s'apparente au troglobie roumain Haplophthalmus tismanicus, est connu de 3 grottes près du v. Lipnica et 3 grottes près de la gare Karlukovo.

Cyphoniscellus gueorguievi est un élément ancien dans la faune bulgare car les deux autres espèces du genre Cyphoniscellus habitent le karst dinarique. $\mathrm{La}$ quatrième espèce ( $C$. bulgaricus) n'est connue que de la grotte Tosova dupka près du v.Glavaci. D'après Vandel $(1967$, p. 350$)$ elle n'est pas proche de C. gueorguievi, mais de C.herzegowinensis. C'est toujours Vandel qui met le genre Cyphoniscellus à la base du système de la sous-fam. de Haplophthalminae.

Revenons à C.gueorguievi. Nous avons trouvé cette espèce en grande quantité dans la plupart des grottes entre le v.Repljana et la colline Pastrinata. Sa 


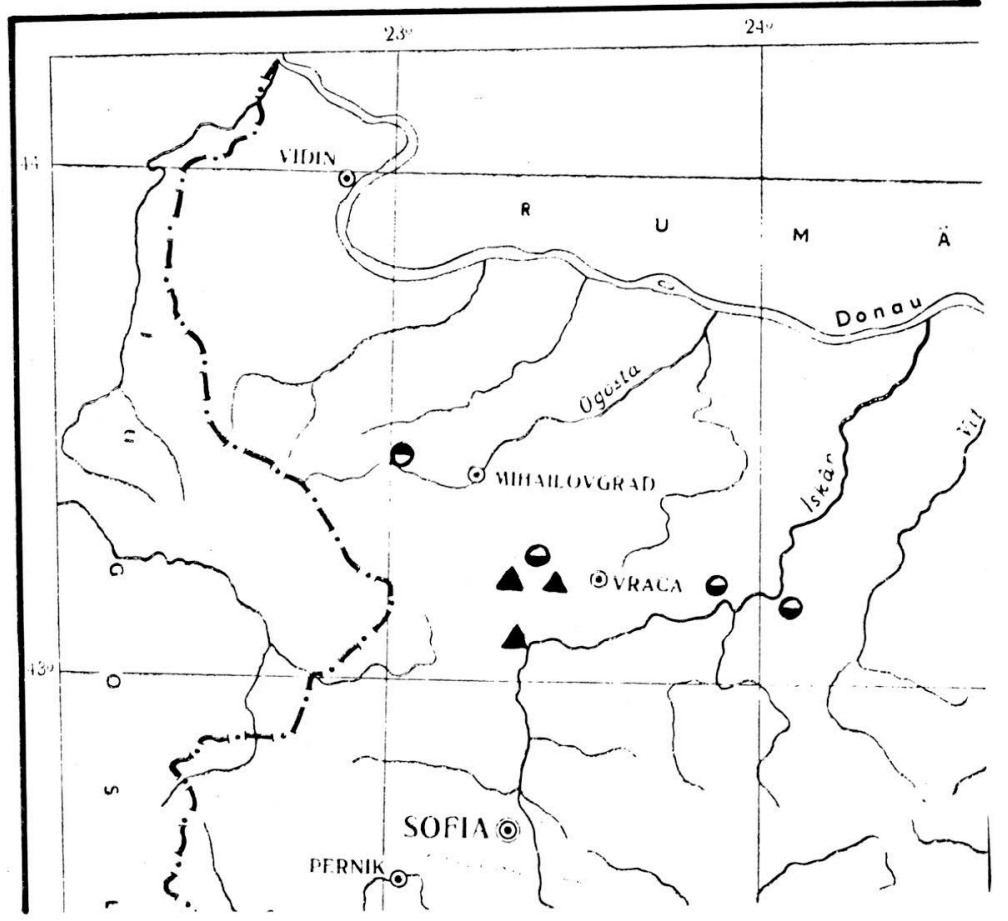

Fig. 4 - $\mathbf{\Delta}$ - répartition de Centromerus bulgarianus $\boldsymbol{\Theta}$ - répartition de Protoleptoneta bulgarica

- répartition de $P$. beroni

répartition serait limitée par la ligne de la transgression tortonienne. Nous ne l'avons pas trouvé, ni dans les grottes près de Cerkaski et Leskovec (dans le golfe de la mer Tortonienne), ni dans les grottes près des villages Bukovec, Smirnenski, Studeno buce, Erden - toutes disposées dans le territoire submergé au tortonien. Les espèces du genre Cyphoniscellus sont vraisemblablement des relictes d'une "Protoégéide du Nord" (Guéorguiev, 1973).

Palpigrada. Nous avons constaté pour la première fois en Bulgarie ces minuscules Arachnides dans les gouffres Meca dupka près de Salas et Randzolova tarsa près de Prevala. Les 3 exemplaires trouvés sont attribués à l'espèce Eukoenenia austriaca. Tous les Palpigrades sont considérés comme des "fossiles vivants" (Janetschek, 1957).

Araneida. Par leur quantité, aussi bien que par le nombre des espèces (33), les Araignées jouent un rôle ạssez important dans les grottes de la Bulgarie du Nord-Ouest. Cependant, du point de vue zoogéographique, leur importance paraît bien inférieure à celle de quelques autres cavernicoles (Diplopodes, Isopodes, Coléoptères). Fait surprenant, il n'y a qu'une seule espèce compléte- 


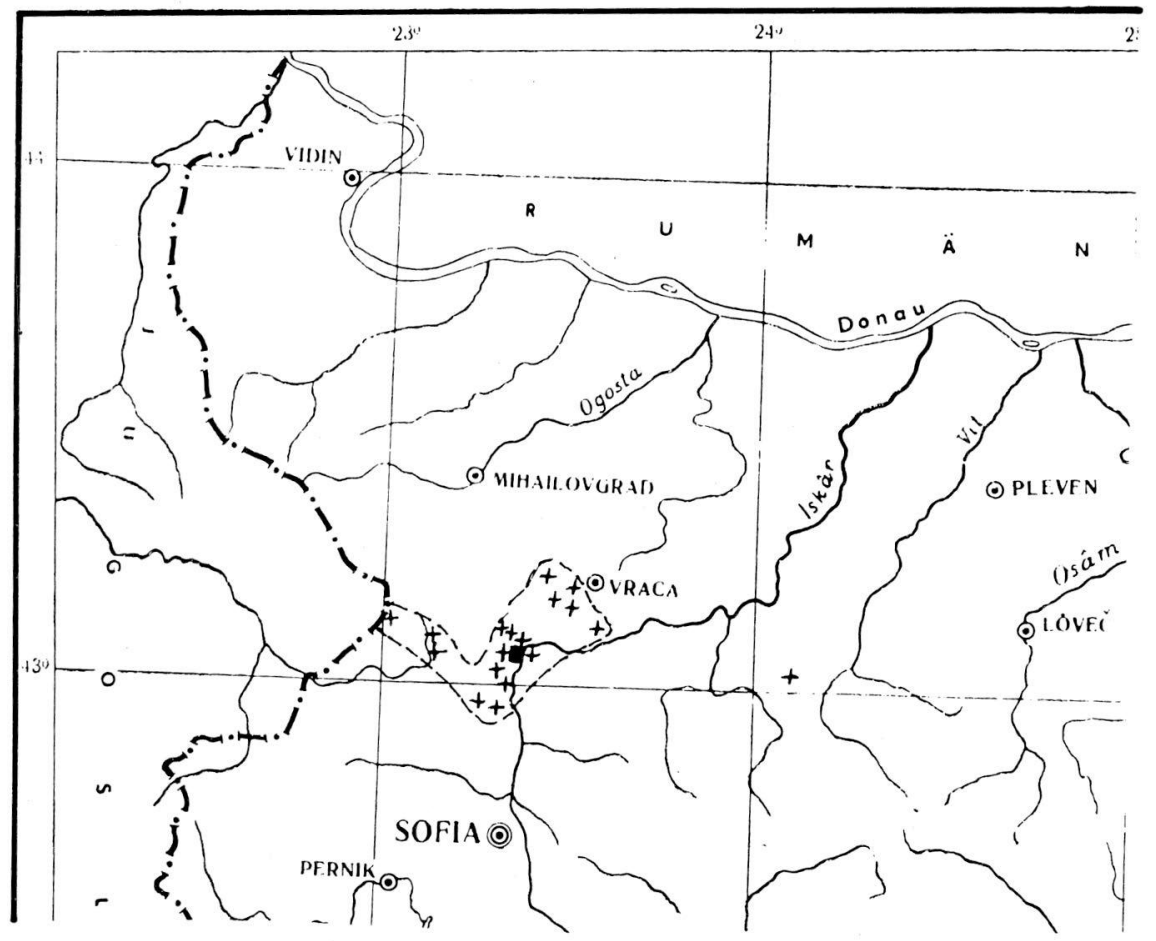

Fig. 5 - + - répartition de Buresiolla bureschi

- répartition de Paralola buresi

ment aveugle parmi les Araignées cavernicoles bulgares (Centromerus bulgarianus), contre 41 Araignées troglobiontes dans les grottes yougoslaves.

La masse des Araignées dans les grottes de la Bulgarie du Nord-Ouest est constituée par quelques espèces troglophiles (Meta menardi, M.merianae, Nesticus cellulanus, 4 espèces du genre Lepthyphantes, Porrhomma convexum, Tegenaria domestica etc.), présentant peu d'intérêt zoogéographique.

On a constaté la présence de 5 espèces endémiques d'Araignées dans la région étudiée (entre Timok et Vit, et notamment:

Lepthyphantes sofianus - connue de 11 grottes. Notons que la Stara planina est habitée par des Lepthyphantes, ayant une répartition plus ou moins large (L.pallidus, L.slivnensis, L.trnovensis, L.leprosus), mais aussi par des espèces (L.sofianus à l'Ouest, L.balcanica dans le Balkan central) aux aires limitées. Ces dernières espèces n'ont pas été trouvées en dehors des grottes. Elles se sont formées dans les régions correspondantes de la Stara planina et nous semblent avoir des exigences assez strictes quant au microclimat des grottes, ce qui explique en partie leurs aires de dispersion restreintes. 


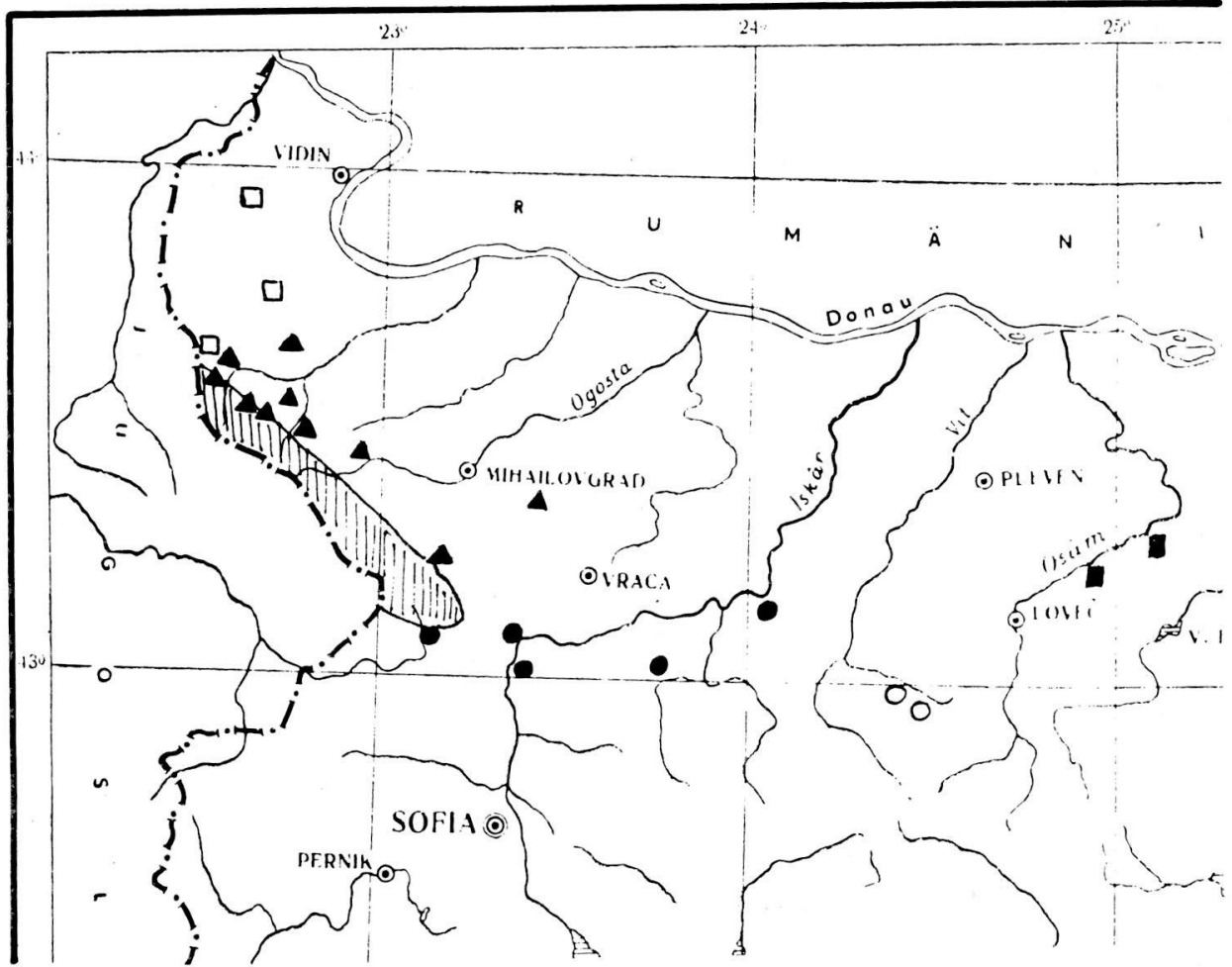

Fig. 6 - - répartition de Typhloiulus bureschi

$\square$ - répartition de T. strictus

$\Delta$ - répartition de Serboiulus spelaeophilus

$\mathrm{O}$ - répartition de $S$. lucifugus deelemani

- répartition de Typhloiulus georgievi

$\boldsymbol{\nabla}$ - répartition de $T$. longipes

- répartition de $T$. staregai

Centromerus lakatnikensis et C.bulgarianus, dont la qualité de "bonnes espèces" a été parfois contestée, sont deux autres représentents de la fam. Linyphiidae, endémiques pour la Stara planina occidentale. C.lakatnikensis est connue de 6 grottes (pas de trouvailles épigées).

Il est intéressant de passer en revue l'histoire des affinités supposées de C.bulgarianus, décrit par Drensky (1931) comme Troglohyphantes bulgarianus et redécrit par Deltchev (1973).

Simon (1911) décrit "Antrobia" europaea d'une grotte des Alpes Maritimes (France).

Fage (1931) communique la même espèce sous le nom de Centromerus europaeus des grottes d'Algérie, d'Espagne et de Lazareva pećina en Serbie orientale.

K ratochvil et Miller (1938) redécrivent l'espèce de Kulczynski Centromerus subcaecus, en la signalant de plusieurs grottes yougoslaves, y compris de Serbie orientale, et considèrent le "Centromerus europaeus", dét. par Fage de Lazareva pećina, soit comme un C.subcaecus aveugle ou 
un C.bulgarianus. Les femelles de ces espèces sont inséparables et c'est le seul sexe connu des grottes de la Serbie orientale.

D'après Decu et Negrea (1969), C.europaeus serait abondant dans certaines grottes d'Olténie. Cette affirmation s'accorde mal avec la liste des Araignées de Roumanie (Fuhn et Oltean, 1970) qui ne fait aucune mention de C.europaeus.

Sans pouvoir donner un avis décisif dans cette discussion, constatons que si C.europaeus habite vraiment les territoires de l'Algérie jusqu'à la Roumanie, ce serait un cas unique parmi les 1600 troglobies terrestres des pays méditerranéens (exception peu probable). Si les exemplaires de la Serbie orientale s'avéraient conspécifiques avec C.subcaecus, ce serait aussi un des rares exemples d'une répartition "transmoravienne" (dans les provinces Dinarique et de la Stara planina à la fois) d'une espèce troglobionte (cas des reliques anciennes). Nous pensons a priori, que toutes ces formes (d'Algérie, des Alpes, d'Herzégovine, de Serbie orientale, de la montagne de Vraca en Bulgarie, de Roumanie etc.) sont proches, mais des taxa distincts, dérivant d'une souche commune largement répandue dans la Mésogéide.

Deux réprésentants du genre Protoleptoneta (fam. Leptonetidae) ont été trouvés par nous dans les grottes de la Stara planina occidentale et décrits par Deltshev sous les noms P.bulgarica et P.beroni. P.bulgarica vit dans 3 grottes entre les petits cailloux humides. Ces localités font partie de la limite septentrionale de la famille dans la région méditerranéenne. Les deux Protoleptoneta sont pour le moment les seules Leptonétidés dans l'Est de la péninsule des Balkans, tandis que l'on connaît dans ses parties occidentales et méridionales aussi des représentants des genres Paraleptoneta et Sulvia. D'après Deltshev (1972), "The genera Leptoneta and Paraleptoneta had been developing as independent phyletic groups and the origin of the two had been Protoleptoneta" (p. 282). Si l'on admet Protoleptoneta bulgarica comme l'espèce la plus primitive de toutes les Leptonétidés de la région Méditerranéenne, il serait facile d'expliquer la présence des Protoleptoneta dans un massif aussi ancien et aussi riche en relictes comme la Stara planina occidentale. Cela corrobore d'ailleurs l'avis de Fage (1913) que le peuplement de la région méditerranéenne en Leptonétidés s'est poursuivi de l'Est vers l'Oüest. La Stara planina occidentale s'avère donc comme un centre important dans l'évolution et la migration des Leptonétidés méditerranéens.

Opilionida. On ne trouve que 2 espèces d'Opilions troglobiontes dans les grottes de la Bulgarie du Nord-Ouest, mais néanmoins elles sont d'un intérêt considérable. Buresiolla bureschi est un troglobie - indicateur pour la région de l'Iskar. Là elle habite plusieurs grottes mais dans la vallée de Botunja elle disparaît brusquement et au Nord on ne la trouve plus. Nous avons trouvé cette espèce dans le gouffre Samak près du v.Komstica (à quelques dizaines de mètres de la frontière avec la Serbie). Atanasov et Stefanov (1951) la signalent de Saeva dupka près de Brestnica, localité bien à côté de l'aire de répartition compacte de B.bureschi (détermination vérifiée par W.Starega). L'autre espèce du genre Buresiolla (B.karamani) habite les grottes de la Bosnie. Il est probable que le genre Buresiolla se soit formé sur l'Egéide Septentrionale avant les transgressions du Miocène.

Relie thermophile, propre des grottes près de la gare Lakatnik, Paralola buresi est un des troglobies les plus remarquables de Bulgarie. Kratochvil (1958) a fondé pour lui une famille à part, Paralolidae, en disant que c'est "eine 


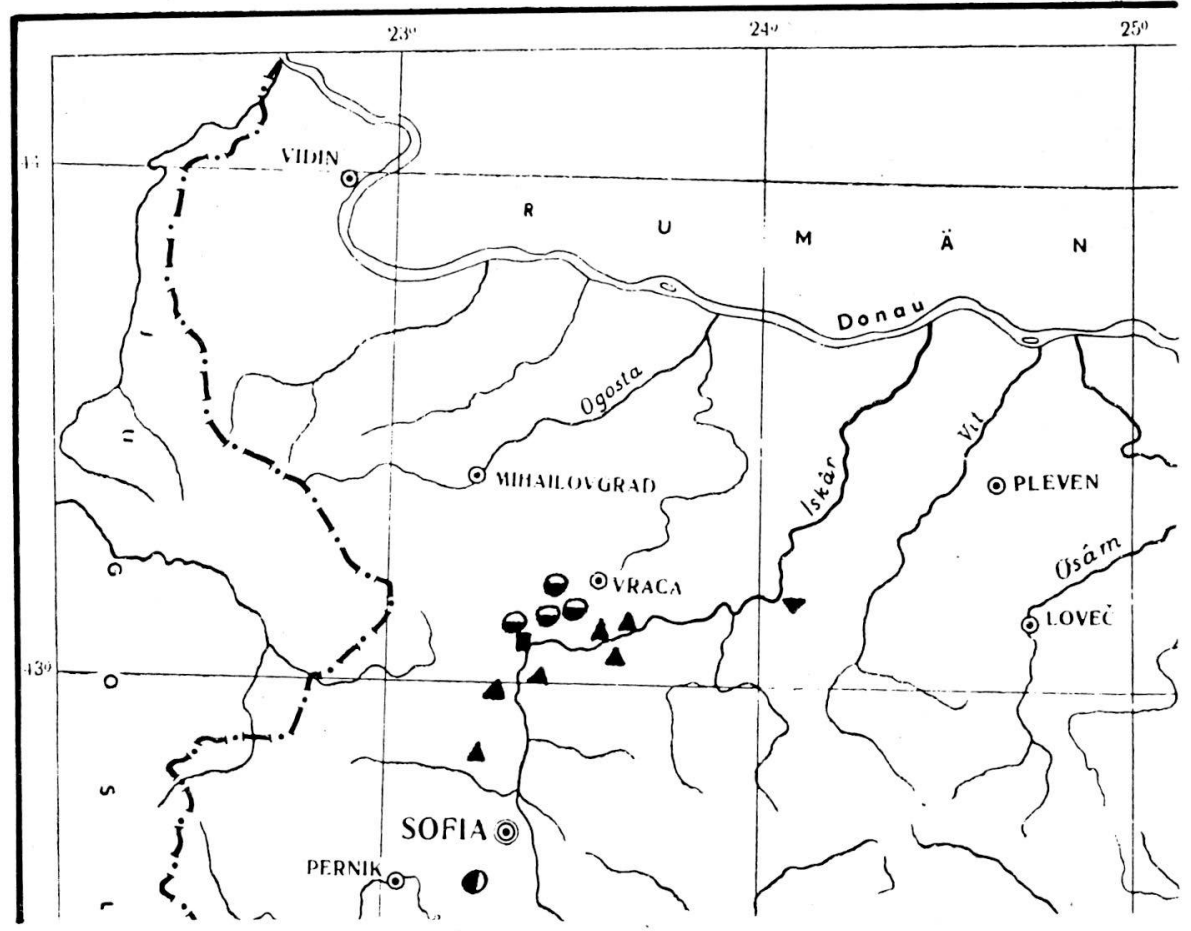

Fig. 7 - - répartition de Bulgarosoma bureschi

O - répartition de $B$. superficiei

- répartition de Trachysphaera orghidani

- répartition de Bulgardicus tranteevi

V - répartition de Brachydesmus radewi

$\Delta$ - répartition de Bacillidesmus bulgaricus bulgaricus

$\boldsymbol{\theta}$ - répartition de $B$. bulgaricus dentatus

$\nabla$ - répartition de Balkanopetalum armatum

äusserst spezialisierte, doch primitive und der Familie Phalangodidae, nahe stehende Weberknechte-gruppe zu sein”, et plus loin: “..ähnliche LaniatoresFormen nicht kennen, erscheinen uns die Paraloliden als isolierte Gruppe der Superfamilie Oncopodoidea, die sich während einer langen Zeit im Raum der östlichen Balkanhalbinsel selbständig entwickelte, vielleicht isoliert von den übrigen Teilen des heutigen Europas" (p. 395). Avec les Oncopodidae d'Asie du Sud-Est, les Paralolidae comptent parmi les Laniatores (pour garder ce nom traditionnel) les plus primitives. La deuxième famille des Laniatores - les Phalangodidés - habite toute l'Europe méridionale (sans la Péninsule des Balkans - une exception pour le genre Lola à l'ile de Hvar), la troisième - les Travuniidae - l'Europe méridionale des Pyrénées jusqu' en Dalmatie. Ces deux dernières familles ne se trouvent pas en Bulgarie. Si l'on considère les Phalangodidés comme étant des relictes mésogéidiennes dans la faune balkanique 
(Kratochvil, 1958, p. 393, écrit même: “..die Vorfahren der Gattung Lola, die eine unzweifelhaft primitivere Form des Sternums als die übrigen europäischen Phalangodiden zeigt, die Balkanhalbinsel bereits in der Vorziet besiedel ten, vielleicht damals, als die Halbinsel noch kein Bestandteil der Mesoägäis war"), il est d'autant plus vrai pour la famille encore plus primitive des Paralolidés.

Outre ces deux troglobies, les grottes de la Bulgarie du Nord-Ouest abritent encore 10 espèces d'Opilions, pour la plupart des trogloxènes. On peut compter parmi les troglophiles Paranemastoma radewi et aussi peut-être Leiobunum rumelicum et au moins un représentant encore non-décrit du genre Siro. Des Cyphophthalmes, Arachnides dont l'origine date du Carbonifère ont été trouvés par nous dans les grottes Hajduskata pestera près du v.Devenci (sous des planches) et Bankovec près du v.Lipnica, sans être jusqu’à présent déterminés spécifiquement. En Yougoslavie ce groupe compte 9 espèces (Hadzi, 1974), mais en Bulgarie on n' a publié que Tranteeva paradoxa de la grotte Rusovata pestera, à l'Est de la rivière Vit (Kratochvil, 1958).

En général, on peut dire qu'il est assez rare de trouver des Opilions dans les grottes de la région d'Ogosta.

Pseudoscorpionida. La péninsule des Balkans est très riche en Pseudoscorpions cavernicoles. Il faut dire néanmoins que le nombre des espèces publiées de sa partie orientale est bien inférieur à celui du karst dinarique. Dans les grottes de la Bulgarie du Nord-Ouest sont représentées les deux familles "classiques" qui renferment la plupart des Pseudoscorpions cavernicoles en Europe: Chthoniidae (le genre Chthonius) et Neobisiidae (les genres Neobisium, Roncus et Acanthocreagris). Nous nous proposons de discuter d'un manière plus détaillée la répartition des Pseudoscorpions cavernicoles bulgares dans une publication ultérieure.

Acaromorpha. Les Acariens terrestres, assez nombreux dans les grottes européennes, peuvent être groupés, à titre provisoire, en quelques catégories, valables aussi pour les Acariens des grottes de la Bulgarie du Nord-Ouest.

1. Parasites obligatoires dans tous leurs stades (sur les Chiroptères, les Rongeurs, les Carnivores, les Arthropodes). Ici on peut classer les familles Sarcoptidae, Myobiidae, Spinturnicidae, Psorergatidae, les Listrophoroidea et autres. Ils passent tout leur cycle de développment sur leur hôte, meurent après sa mort et n'ont pas (pratiquement) de relations avec le milieu souterrain.

2. Parasites ayant certains stades parasitaires (ou commensaux) tandis que d'autres stades en sont libres. Telles sont les Trombiculidae (les larves parasites surtout des Vertébrés), les Trombidiidae (larves parasites des Invertébrés, les nymphes et les adultes des deux familles sont libres), certains Acaridae et Glycyphagidae (deutonymphes ou hypopes phorétiques, adultes libres), Laelapidae (deutonymphes ou hypopes phorétiques, adultes libres), Laelapidae s. lato (chez la plupart tous les stades sont des parasites ou des commensaux, mais on les trouve parfois sur le guano ou autres substrats dans les grottes). Les Ixodidae et Argasidae entrent aussi dans cette catégorie - leur larves, nymphes ou femelles adultes sont des parasites permanents, alors que les mâles sont vus souvent rampant sur le plancher ou la paroi des grottes.

3. Saprophages - habitent le guano, le bois pourri etc. à tous les stades. Tels sont certains Tyroglyphoides, les Oribates et autres.

4. Prédateurs - habitent les mêmes substrats, mais sont voraces. Exemples: la plupart des Prostigmata libres (cunaxidae, Rhagidiidae, Bdellidae, Nicoletiellidae).

C'est assez difficile d'intercaller les Acariens habitant les grottes dans le schéma géneral du groupement écologique des cavernicoles. On a appelé cer- 


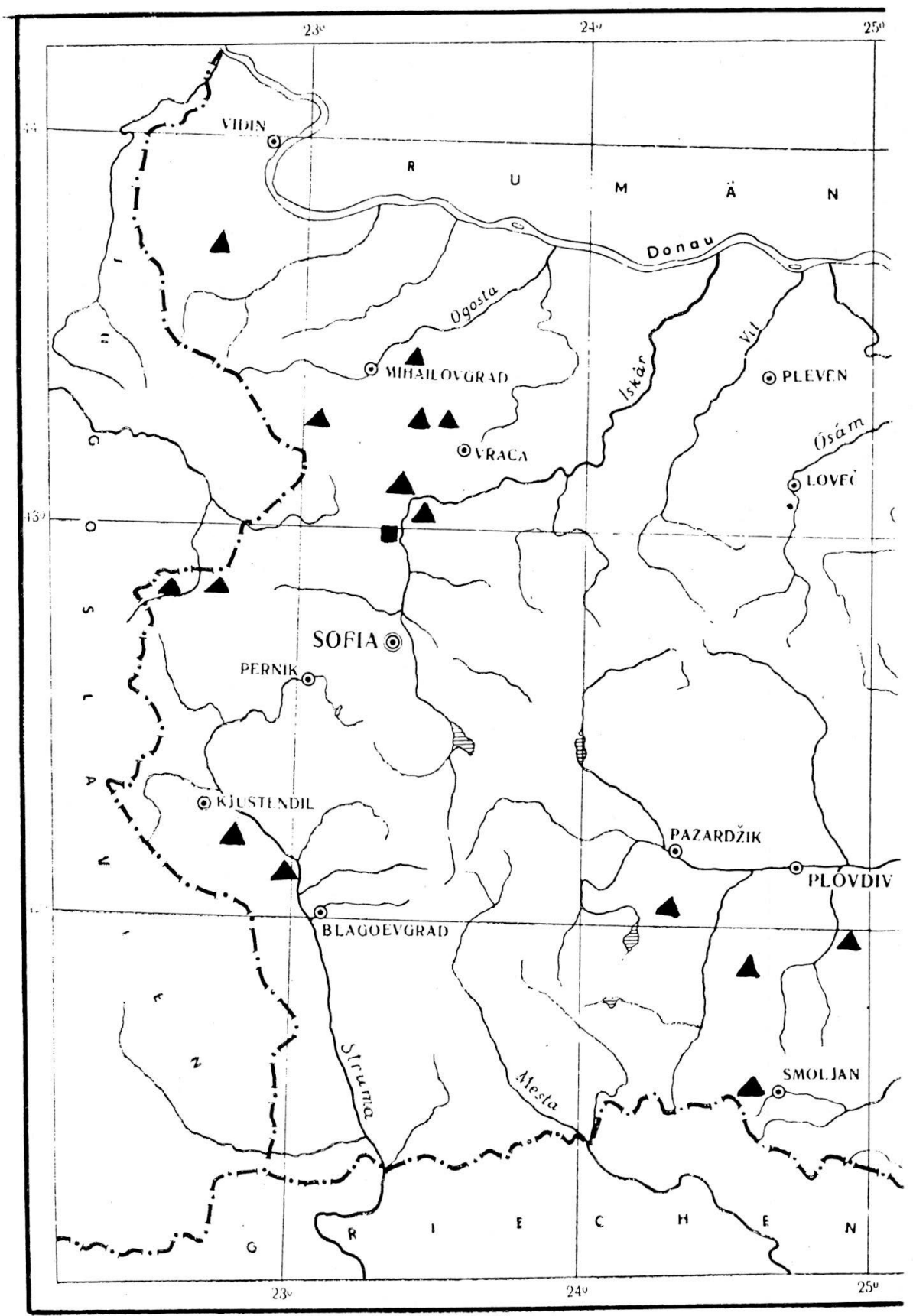


taines espèces parfois des troglobies, parfois des troglophiles. D'autres Acariens habitent aussi bien les grottes que l'hypolithon ou l'hypoxylon (ex.: Eupodidae). Des Acariens comme les Rhagidiidae semblent être préadaptés à une vie dans le domaine souterrain, mais leur écologie demeure peu connue.

Jeannel (1926) dit: "Les seuls Acari que l'on puisse dire troglobies sont les Rhagidia" (p. 161).

Dans sa monographie sur la faune cavernicole de Belgique Leruth (1939) qualifie comme troglobie parmi les Acariens terrestres seulement Schwiebea cavernicola (que l'on trouve aussi dans les grottes de la Bulgarie du NordOuest), tout en considérant les Rhagidia comme troglophiles. Willmann (1941) définit Rhagidia strasseri comme troglobie et les autres Rhagidia comme plus ou moins troglophiles.

La qualification d'Ixodes vespertilionis varie d'après les auteurs de "trogloxène" à "troglophile" même jusqu'à "troglobie" (Vandel, 1964). Une analyse assez détaillée, aussi bien que des objections contre sa qualification de "troglobie" se trouve dans l'ouvrage de Beaucournu (1967). Dusbábek (1972) a classé parmi les troglobies les espèces I.vespertilionis et I.simplex et aussi 3 espèces de Trombiculidae - toutes les 5 espèces vivent également dans les grottes de la Bulgarie du Nord-Ouest.

Pour le moment nous hésitons à considérer comme troglobies les Acariens peuplant les grottes bulgares.

Diplopoda. Le territoire entre Timok et Vit compte 8 Diplopodes troglobies parmi sa faune cavernicole - c'est la moitié de tous les troglobies bulgares de ce groupe. Leur répartition est assez remarquable, d'autant plus que, d'après certains auteurs, un bon nombre des Diplopodes cavernicoles d'Europe sont des représentants d'anciennes lignées mésogéidiennes, ayant une grande valeur zoogéographique.

Les grottes de la Bulgarie du Nord-Ouest sont caractérisées par une prédominance bien marquée des Typhloiulini. D'aprés Tabacaru (1970), sur le territoire de l'Egéide Septentrionale ils se substituent aux Blaniulidés, propres à la Tyrrhénide. En Bulgarie Orientale et Méridionale on n'a pas trouvé des Typhloiulini troglobies, mais là ils sont "remplacés" par les Apfelbeckiellini.

Tabacaru est d'avis que le centre principal de l'evolution des Typhloiulini s'est situé aux Alpes du Sud-Est, d'où ils se sont répartis à travers les Dinarides, en atteignant la Stara planina orientale (Typhloiulus kotelensis qui n'est pas troglobie) et le Péloponèse (Typhloiulus sp. - un troglobie non encore décrit, fide Strasser, 1974). Cette migration date d'une époque reculée - rappelonsnous que le Sillon transégéen a existé du Paléogène jusqu' au début du Pliocène. Les aires de dispersion des divers Typhloiulini (Typhloiulus et Serboiulus) en Bulgarie du Nord-Ouest se sont formées d'une manière assez curieuse.

Les deux Serboiulus (S.lucifugus de Serbie Orientale et $S$. spelaeophilus des grottes de la région d'Ogosta) ont des aires géographiques voisines, quoique séparées par la chaîne des Balkans, du Todorini kukli jusque’à Midzur. Les

Fig. 8 - $\Delta$ - répartition de Lithobius lakatnicensis

$\square$ - répartition de Eupolybotrus andreevi 


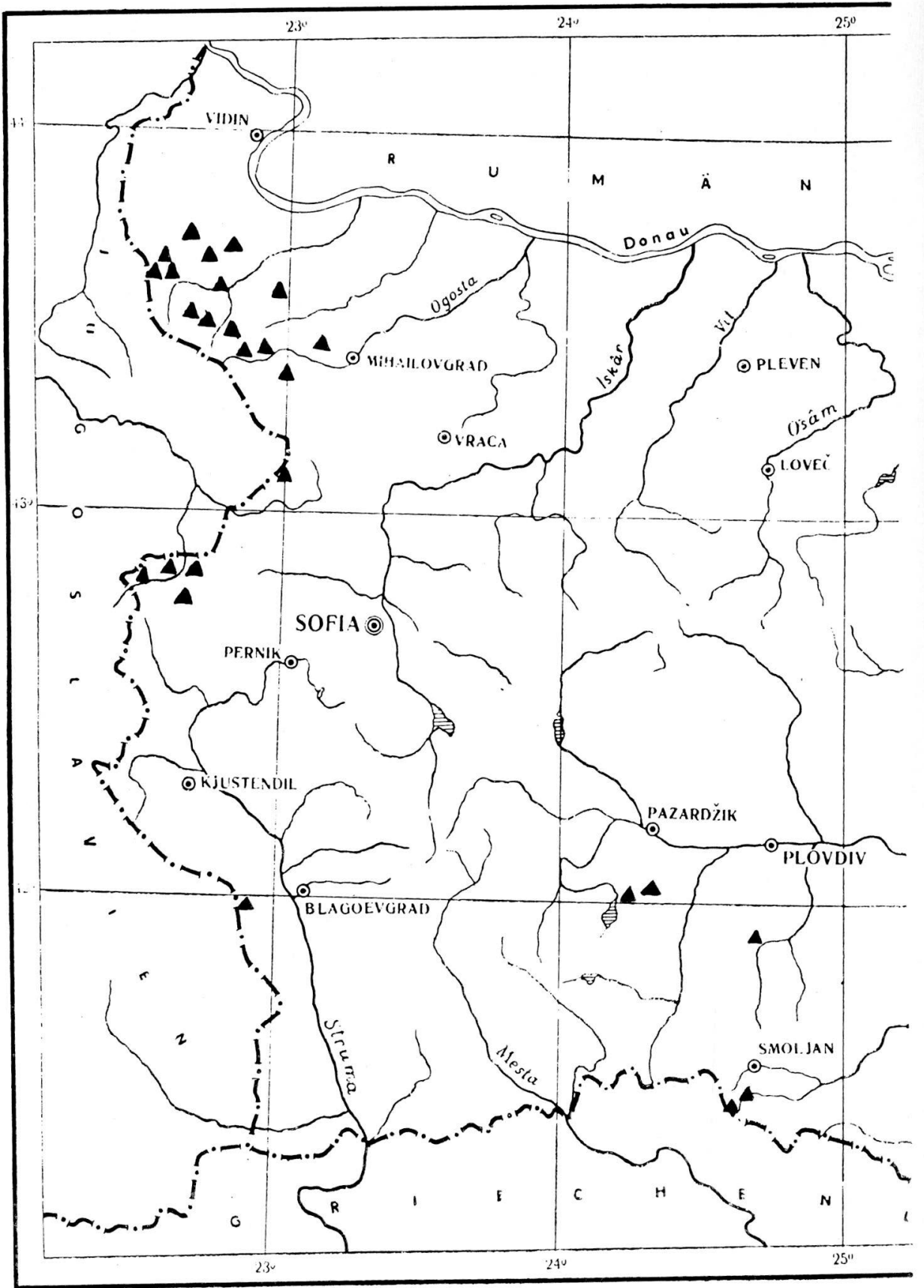


Serboiulus sont entourés par les aires de repartition des espèces du genre Typhloiulus. A notre avis, il est bien probable que Serboiulus soit un genre plus récent, dérivé du Typhloiulus. La proche parenté des deux genres fut confirmée indirectement par Golemansky, qui a établi l'existence de Grégarinides identiques dans l'intestin de T.bureschi et de S.spelaeophilus (Stenophora typhloiuli). L'aire de répartition de Serboiulus spelaeophilus se trouve en dehors de la transgression Tortonienne. On n'a trouvé des Diplopodes troglobiontes au Nord de Belogradcik et de Salas. Ils font défaut dans les grottes de Rabiska mogila et dans les grottes du territoire, ayant été submergé par la transgression Tortonienne. Il est évident que ces espèces ont peuplé le domaine souterrain à une époque antérieure au Tortonien. Fait curieux, l'unique localité du Serboiulus au Nord de la transgression Tortonienne est située sur une île de la mer Tortonienne (la grotte Kopanata dupka près du v.Portitovci, distr. de Mihailovgrad)! Les grottes au Nord de la ligne Belogradcik - Salas sont peuplées par le Typhloiulus strictus (troglophile), connu aussi de la Serbie orientale et du Banat.

Une localité aberrante de Serboiulus spelaeophilus, c'est la galerie artificielle près de la gare Thompson. Cette trouvaille nous est difficile à expliquer pour le moment.

Un "noeud" où se rejoignent les aires de dispersion de Typhloiulus bureschi, Serboiulus spelaeophilus et S.lucifugus deelemani, c'est la région de Kalotina - Komstica. Jusqu'à présent nous n'avons pas réussi à trouver de Diplopodes troglobies dans une dizaine de grottes prospectées dans cette région.

Fait intéressant et qui, à notre connaissance, n'est pas mentionné pour les Diplopodes troglobies, c'est l'existence de localités isolées de certains Typhloiulini (par exemple, Typhloiulus longipes, T.staregai) sur la périphérie de l'aire compacte d'autres espèces de la même tribu. $\mathrm{Il}$ nous semble de règle que l'on ne trouve dans une grotte qu'une seule espèce de Typhloiulini. La seule exception, c'est la cohabitation de Typhloiulus staregai (peut être un troglophile?) et de Serboiulus spelaeophilus dans la grotte Prelaz (Ruskovica) près du v.Salas, cas unique de cohabitation d'un Typhloiulus et d'un Serboiulus. Chez les troglobies Typhloiulus bureschi et Serboiulus spelaeophilus la ligne de démarcation est assez stricte. Nous l'avons établie là où se terminent les aires de dispersion des autres troglobies caractéristiques pour la montagne de V raca: les Pheggomisetes, Buresiolla bureschi, les Paraduvalius, Rambousekiella ledenikensis, Bureschia bulgarica etc. C'est le terrain entre les villages Dolno Ozirovo et Botunja. Il est hors de doute que cette limite si brusque a été déterminée par des causes géologiques (paléogéographiques et tectoniques).

La répartition des Antroleucosomatidés n'est pas moins intéressante. Bulgarosoma bureschi paraît cantonnée sur une aire restreinte dans la montagne de Vraca. On ne trouve cette espèce, ni dans les grottes du défilé de l'Iskar, ni au Nord-Ouest de Botunja. Les autres espèces du genre Bulgarosoma habitent les grottes de la Serbie orientale, du Banat et du R hodope. Leurs migrations ont eu lieu avant la transgression Tortonienne. Du même avis est aussi Ta-

Fig. 9 - $\mathbf{\Delta}$ - répartition de Troglophilus neglectus 


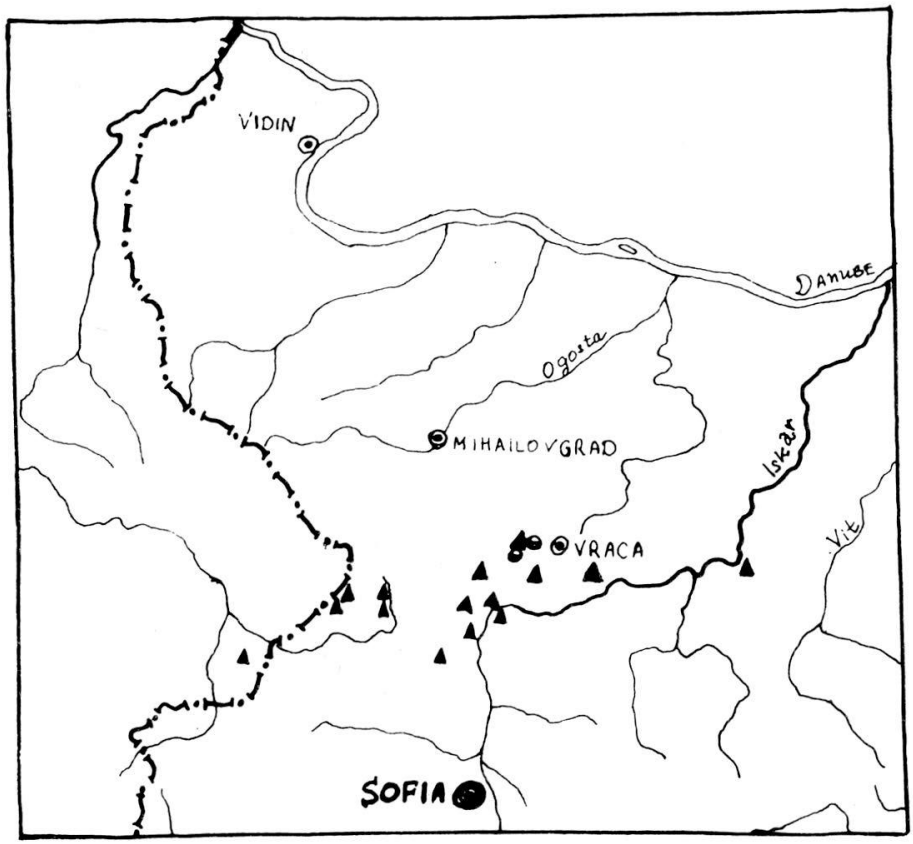

Fig. 10 - $\mathbf{\Delta}$ - répartition du genre Pheggomisetes

- répartition de Rambousekiella ledenicensis

bacaru (1970) concerant l'espèce roumaine B.ocellata. D'autre part, le district de Smoljan où habite Bulgarosoma meridionale, a été submergé pendant l'Eocène supérieur (Boncev, 1971, fig. 31).

La localisation actuelle des Bulgarosoma est conditionnée par un préférendum écologique assez strict. Il nous semble que les espèces de ce genre préfèrent les grottes du Rhodope, ayant une température relativement basse, et les parties élevées de la montagne de Vraca. On n'a pas observé une cohabitation des Typhloiulus et des Bulgarosoma dans une même grotte, mais dans la grotte Erkjuprija près du v. Mostovo (Rhodope) Bulgarosoma rhodopinum vit avec Apfelbeckiella beroni beroni.

Tout récemment nous avons trouvé (sous pierre) à Vitosa, près du châlet Aleko, à 1950 m d'alt., un exemplaire du genre Bulgarosoma. Strasser (1975) a décrit, en se basant sur cet exemplaire, la première espèce épigée (quoique aveugle!) du genre Bulgarosoma: B.superficiei. C'est un cas plutôt rare parmi les Diplopodes, bien que chez les Coléoptères et quelques autres groupes on connaisse certaines expèces d'aspect "troglobionte", vivant sous les pierres à une altitude d'env. 1500-2000m.

Parmi les Trachysphaera des grottes de la Stara planina il n'y a qu'une seule espèce troglobie: T.orghidani (sous-espèce encore inèdite de l'espèce habitant 
les grottes des Carpates roumanines). C'est le seul cas d'espèce commune entre les faunes troglobiontes de la Stara planina et des Carpates. Les Trachysphaera sont consdiérrées être des relictes mésogéidiennes dans la faune cavernicole d'Europe - leurs espèces troglobies sont répandues des monts Cantabriques jusqu'à Dobroudza. Nous n'avons pas trouvé plus d'une seule espèce de ce genre dans une même grotte.

Brachydesmus radewi et Bulgardicus tranteevi net sont connues que d'une seule grotte chacune.

Parmi les Polydesmidiens, le troglophile le plus répandu dans les grottes de la Stara planina est le Polydesmus renschi.

Nous avons récolté en grande quantité sur le guano de la grotte Desni Suhi pec près du v.Dolni Lom un mince Diplopode blanchâtre que Strasser (1962) a décrit sous le nom de Bacillidesmus bulgaricus, en le rangeant d'abord parmi les troglophiles, mais ensuite, et d'après nous avec raison, parmi les troglobies. Avec sa sous-espèce dentatus, B.bulgaricus paraît propre à la Bulgarie du Nord-Ouest. La deuxième espèce du genre - B.filiformis - est un endogé de Vojvodina (Serbie). D'après Verhoeff (1941, p. 180), tout le groupe Trichopolydesmidae - Bacillidesmidae est d'origine américaine. Cet auteur émet l'hypothèse peu probable que pendant le Tertiaire ce groupe s'est répandu par l'intermédiaire d'une "Atlantide" de l'A mérique du Nord en Europe, où l'on trouve maintenant ses decendants. Il faut dire que l'existence d'une "Atlantide", ou d'un autre pont continental, liant l'Europe à l'Amérique du Nord est contestée, niée même, par plusieurs auteurs. On peut penser plutôt à une autre possibilité: les souches communes des Bacillidesmidiens et des formes qui leurs sont proches (Trichopolydesmus, Verhoeffodesmus, Galliocookia et les lignées américaines) auraient peuplé la Laurasie avant le Jurassique. Tabacaru (1970) est aussi d'avis que ces représentants de l'ordre Proterospermophora sont des relictes très anciens dans la faune d'Europe.

Nopoiulus venustus que l'on trouve dans plusieurs grottes et galeries artificielles de la Stara planina, est connue aussi d'autres pays d'Europe. Chez nous on peut la considérer comme troglophile.

Le genre Balkanopetalum, endémique pour la faune bulgare, est représenté par l'espèce (B.armatum) dans les grottes et les galeries artificielles de la Bulgarie du Nord-Ouest et par 2 autres espèces (B.rhodopinum et B.beshkovi) dans les grottes du R hodope. Toutes les trois sont des troglophiles. Golemansky (in litt.) a constaté qu'une même espèce de Grégarine (Stenophora beroni) vit dans l'intestin de l'espèce de la Stara planina B.armatum et de l'espèce du Rhodope B.rhodopinum.

Chilopoda. Aussi bien par le petit nombre des troglobies que par sa qualité d'indicateurs zoogéographiques, les Chilopodes sont un groupe dont l'importance est de loin inférieure à celle des Diplopodes. En Bulgarie du Nord-Ouest on en connaît 2 troglobies. La répartition du premier - Lithobius lakatnicenses - est quelque peu singulière: la Serbie orientale, la Stara planina du NordOuest, les grottes près de Tran et de Kjustendil, le Rhodope. Cette répartition indique une colonisation ancienne du domaine souterrain, mais en même temps cette colonisation a du avoir lieu après l'Oligocène, car à cette sous-époque 


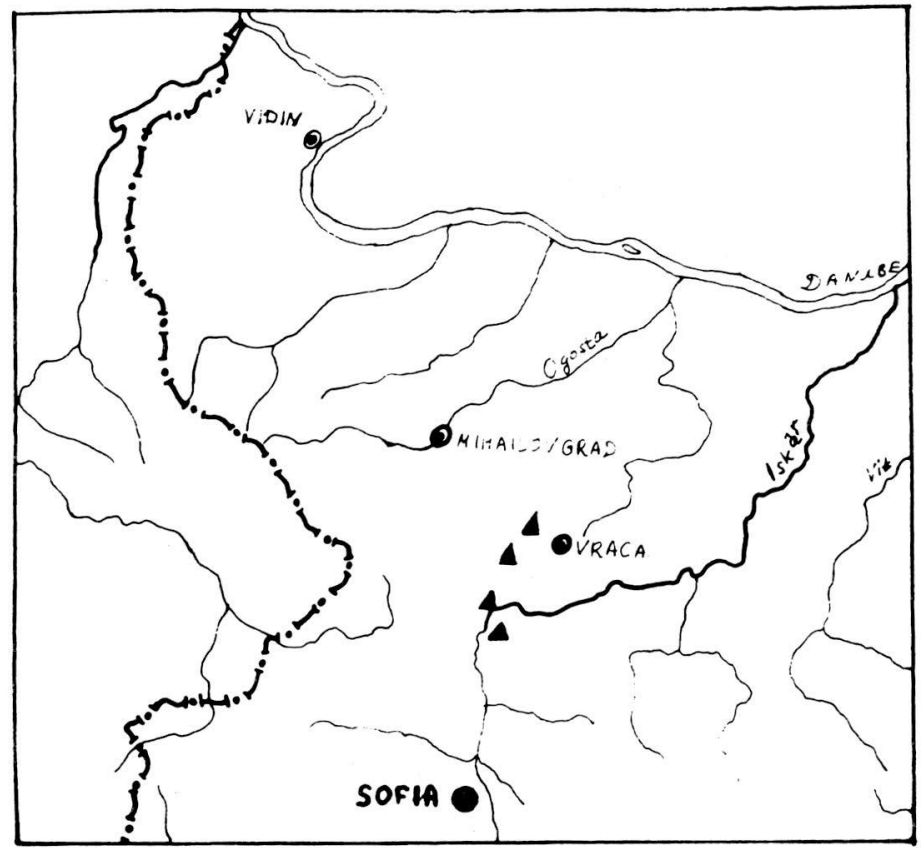

Fig. 11 - $\Delta$ - répartition de Duvalius (Paraduvalius) en Bulgarie du Nord-Ouest

certaines localités en Bulgarie du Sud sont encore submergées (la grotte Uske près du v. Cetirci, distr. Kjustendil).

Eupolybothrus andreevi, Chilopode de grande taille que Matic a décrit de la grotte Vodnata pestera près de la gare Cerovo, n'est connue que de cette localité. Avec l'Eupolybothrus obrovensis de Yougoslavie, ce sont les deux seules espèces aveugles de ce genre.

Collembola. On a trouvé jusqu'à présent 35 espèces de Collemboles dans les grottes bulgares. En comparant ce nombre avec les espéces (plus de 80) connues des grottes roumaines, on peut conclure qu'il nous reste beaucoup à faire pour avoir une idée exacte concernant les Collemboles cavernicoles bulgares.

La plupart de ces 35 espèces (28) ont été récoltées dans les grottes de la Bulgarie du Nord-Ouest. Certaines d'entre elles sont largement répandues dans les grottes d'Europe (Mesogastrura oicoviensis, Ceratophysella denticulata, Heteromurus nitidus, Arrhopalites pygmaeus, Tomocerus flavescens etc.). Gruia (1969) considère comme étant d'origine méditerranéenne Onychiurus postumicus, O.subgranulosus et autres. Si nous regardons de plus près les aires de dispersion de quelques Onychiurus tenus pour des troglobies, nous les trouverons certainement assez inhabituelles pour des expèces de cette catégorie: O.postumicus - Istrie, Roumanie, Stara planina, R hodope; O.vornatscheri 
- Autriche, Roumanie, Bulgarie. Vandel(1964) considère les Onychiurus comme étant des troglobies récents, liés plutôt au milieu souterrain au sens large qu' aux grottes.

Certaines espèce de ce genre, malgré leur aspect d'animaux mal protégés, semble être assez résistantes au froid et aux autres paramétres extrêmes du milieu (nous les avons vu dans les grottes-glacières et aussi dans les racines des succulents près du refuge de Goûté aux Alpes, à $3800 \mathrm{~m}$ d'alt.). Nous qualifierons de troglobies aussi O.beroni des grottes près de la gare Oresec et $\mathrm{O}$. bureschi et O.sensitivus de la grotte Ledenika près de Vraca. Gruia (1969, p. 169) admet que cette dernière espèce est proche de l'Onychiurus orghidani des Carpates roumaines. Dans sa monographie sur la faune cavernicole de la Suisse Strinati (1966) énumère 24 espèces troglobiontes parmi les Collemboles de ce pays (y compris Pseudosinella duodecimocellata et Arrhopalites pygmaeus que l'on trouve aussi en Bulgarie). Nous ne pouvons pas, d'après nos critères actuels, classer ces deux espèces parmi les troglobies et en général nous pensons que le nombre de Collemboles troglobies suisses (24) n'est par réel (surtout si on le compare avec le nombre des troglobies de ce groupe dans un pays plus grand et assez bien étudié comme la Roumanie lequel n'est que de 8).

Nos observations sur les Collemboles flottant sur la membrane superficielle des flaques dans les grottes bulgares confirment la constation de Botosaneanu (1971) que: "pour une fraction de ces espèces la pellicule superficielle de l'eau ne représente pas tout simplement un "piège", mais bien un milieu d'élection".

Diplura. Le matériel de Diploures cavernicoles de la Stara planina est demeuré pour la plupart indéterminé. Plusiocampa bulgarica fut trouvé aussi bien dans les grottes de la Stara planina que dans celles du Rhodope. Il reste a préciser la position taxonomique des espèces, habitant les grottes de Lakatnik (Plusiocampa bureschi, P.rauseri) et la grotte Magura. Si P.bulgarica est en effet une espèce complexe (comme c'est l'avis de Codé, in litt.), la présence de cette "espèce" à la fois dans les grottes de la Stara planina et du Rhodope deviendra moins énigmatique.

On peut noter que, selon nos observations, les Diploures sont nombreux dans les grottes de la région de l'Iskar et surtout dans les grottes du Rhodope, tandis que dans les grottes de la région d'Ogosta on ne les trouve que très rarement.

Orthoptera. La répartition des Orthoptères cavernicoles en Bulgarie est assez particulière. Le matériel n'étant pas étudié en détail, nous considérons tous les exemplaires récoltés dans les grottes bulgares comme appartenant à une seule espèce: Troglophilus neglectus. Son aire de dispersion, englobe la Yougoslavie, l'Autriche du Sud et la Grèce du Nord. Chez nous on l'a trouvé dans plusieurs grottes le long de la frontière bulgaro-yougoslave et dans le Rhodope. En dehors de T.neglectus, on connaît en Yougoslavie 5 autres expèces du genre Troglophilus et 3 espèces des genres Dolichopoda et Gryllomorpha.

La limite orientale de l'aire de dispersion de T. neglectus passe par la Bulgarie. C'est un fait curieux que lá où ce Raphidophoridae se trouve, il habite toutes les grottes, grandes et petites, présentant des conditions microclimatiques assez variées. Plus loin on trouve aussi des grottes, mais aucune n'est peuplée par des 


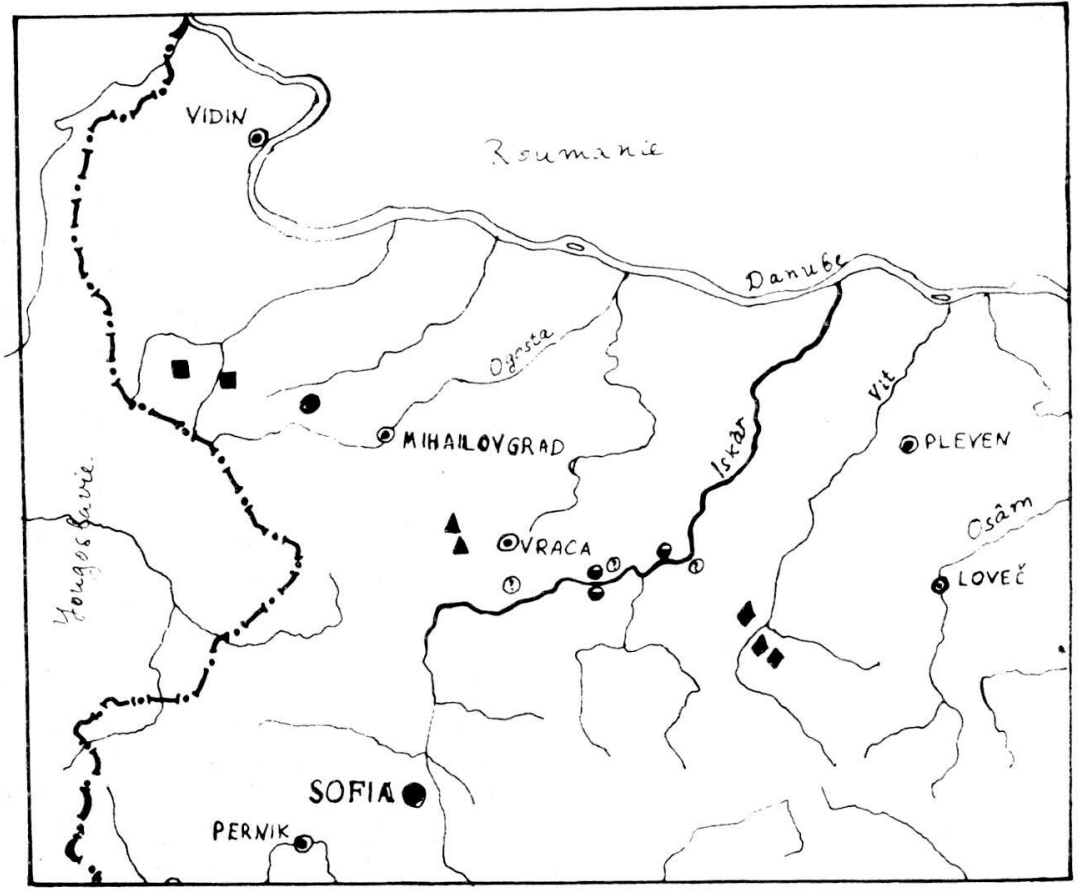

Fig. 12 - - répartition du genre Beronia

O - répartition du genre Beskovia

A - répartition du genre Radevia

(3) - répartition des Bathysciinae non-déterminés jusqu'au genre

- répartition du genre Tranteeviela

Troglophilus. Dans les grottes de la Stara planina on n'a pas vu de mâles de cette espèce.

Quoique non troglobies, les Raphidophoridae sont qualifiés de relictes dans la faune européenne. Jeannel les considère comme étant des dérivés de lignées gondwaniennes, s’étant développées sur les terres de la Méditerranée avant la transgression lutétienne. Il est aussi intéressant d'indiquer le fait de l'existence de plusieurs espèces de ce groupe dans les parties occidentale et méridionale de la péninsule du Balkan, alors qu'une montagne aussi riche en troglobies que la Stara planina à l'Est de Mihajlovgrad est totalement dépourvue d Orthoptères caverernicoles.

Coleoptera. Nous avons constaté la présence de 9 familles de Coléoptères dans les grottes entre Timok et Vit. Il n'y a que 2 d'entre elles (Carabidae - Rambousekiella, Pheggomisetes et Duvalius - et Catopidae) qui contiennent des troglobies.

Du seul représentant des Pterostichinae (Rambousekiella ledenikensis) on n'a trouvé que 3 exemplaires dans 3 grottes de la montagne de Vraca. Peut-être 
ce n'est pas du tout un vrai cavernicole, mais plutôt un habitant des fentes profondes. Après la capture de l'holotype de cette espèce dans la grotte Ledenika en 1924, cela fait plus d'un demi-siècle que l'on cherche en vain (y compris en mettant des appâts) une deuxième Rambousekiella dans cette grotte.

L'aire de dispersion des Pheggomisetes est limité par le trapézoïde Komstica - Karlukovo - Cerovo - Odorovci. Ce genre particulier a été considéré par Laneyrie (1970) comme membre unique d'une lignée phylétique proche des Aphaenops et des Neotrechus. On n'a pas trouvé en Bulgarie de représentants de ces deux séries, dont les espèces sont répandues de l'Espagne, resp. de l'Italie jusqu'au Caucase. Les Pheggomisetes sont une lignée isolée d'origine mésogéidienne, peut-être dérivant d'une souche aphénopsienne du Paléogène.

Chez les Pheggomisetes on observe le phénomène assez rare parmi les troglobies - cohabitation de deux espèces appartenant à un même genre dans une même grotte (Ph. buresi et Ph.radevi dans la grotte Medenik; Ph. buresi et Ph.globiceps dans la grotte Goljamata meca dupka - voir Guéorguiev, 1964). Ph.radevi est bien plus rare que Ph.buresi (trouvé par exemplaires isolés). Les Pheggomisetes sont des indicateurs excellents de la région de l'Iskar. La plupart de leurs localités se trouve à l'Ouest de l'Iskar, mais on a constaté aussi deux localités à l'Est de cette rivière: Ph.globiceps georgievi dans le gouffre Jamata près du v.Lakatnik et Ph.globiceps karlukovensis d'un gouffre près du v.Karlukovo, Si le statut taxonomique des sous-espèces des Pheggomisetes n'est pas altéré par une nouvelle révision, basée sur l'étude statistique de grandes séries, nous aurons devant nous une mosaïque de populations - chose inhabituelle pour les troglobies, aussi bien que pour les autres genres de la sous-famille des Trechinae.

Le troisième genre de cette sous-famille - Duvalius - est représenté dans les grottes de la Bulgarie du Nord-Ouest par 4 espèces aveugles du sous-genre Paraduvalius. L'aire de dispersion de ce sous-genre englobe la Stara planina, la Serbie orientale, la Macédoine et le Rhodope (les provinces biospéléologiques de la Stara planina et du Rhodope). Il est bien significatif que nous n'ayons trouvé aucun Paraduvalius dans les quelque 130 grottes soigneusement étudiées au Nord-Ouest de la rivière Botunja, tandis que la Montagne de Vraca en abrite au moins 3 espèces. Ce fait plaide une fois de plus en faveur de l'idée de l'existence d'une barrière qui ait empêché les Paraduvalius, les Pheggomisetes et un bon nombre d'autres troglobies d'envahir les grottes de la région d'Ogosta. C'est d'autant plus curieux, étant donné que les Paraduvalius peuplent toute la Stara planina (jusqu'à Kotel), aussi bien que la Serbie orientale. Il est évident que la dépression de Vit ne constitue aucune barrière pour eux. Alors qu'en Macédoine on trouve aussi des Paraduvalius endogés, les espèces de Stara planina et la seule connue jusqu'à présent espèce du Rhodope $(D$. (P.) bureschi) ne vivent que dans les grottes. Ce sous-genre paraît d'origine nord-égéïdienne, tandis que pour les Duvalius (s.str.), répandus de l'Espagne jusqu'à la Chine. Jeannel (1928) suppose une origine sud-égéïdienne. Parfois on considère la série phylétique des Duvalius comme une série de troglobies bien plus récents que les Pheggomisetes, mais la grande étendue de leurs aires de dispersion et surtout la présence du genre Ameroduvalius en 
Amérique nous incline à penser que les Duvalius sont plutôt úne série ancienne. Prenant en considération que les Duvalius se répandent relativement vite, on peut admetre le sous-genre Paraduvalius comme de type néoendémique.

STAPHYLINIDAE. Des 14 espèses de cette famille vivant dans les grottes en Bulgarie du Nord-Ouest, les plus importantes nous semblent être Quedius mesomelinus et Atheta macroptera - habitants du guano de Chauves-souris et des excréments de Renards et de Blairaux. Comme endémiques pour ce territoire nous avons Quedius troglophilus et Q. gueorguievi.

HISTERIADAE. Deux grottes à guano (Mitrevska dupka près du v. Gorna Luka et Prilepnata pestera près de Liljace) abritent Gnathoncus rotundatus et G. nannetensis, espèces peut-être troglophiles. Les Gnathoncus fréquentent aussi la grotte Bahardénskaja pestera en Asie centrale soviétique (Kuzjakin, 1950).

PSELAPHIDAE. Bryaxis beroni est la seule espèce de cette famille connue des grottes bulgares. Elle n'a été trouvée que dans 4 grottes, toutes dans la Bulgarie du Nord-Ouest. Nous pensons que c'est un troglophile.

Les CATOPIDAE troglobies en Bulgarie appartiennent tous à la sous-fam. des Bathysciinae. D'après la classification récente de Guéorguiev (1974), tous les 7 genres de ce groupe, habitant les grottes de la Stara planina, entrent dans la tribu Bathysciini, et notamment: Beronia et Radevia - sous-tribu Pholeuonina, Netolitzkya et Hexaurus - Bathysciina; Beskovia, Balcanobius et Tranteeviella - Leptodirina. Des plus de 130 grottes prospectées dans la région d'Ogosta, nous n'avons trouvé des Bathysciinae que dans 7, dont seulement Beronia micevi des grottes près de Belogradcik a été déterminée spécifiquement. Quant aux autres genres de cette sous-famille, trouvés dans la Stara planina occidentale, on peut dire que les Beskovia habitent les grottes de faible altitude (200-300 m) le long de la rivière Iskar, tandis que les Radevia sont propres plutôt aux grottes de la montagne de Vrace (800-900 m).

D'après le schéma de Guéorguiev (1974) les Bathysciina et les Pholeuonina sont d'origine mésogéidienne, alors que les Leptodirina sont considérés être des descendents des Pholeuonina, ayant une origine nord-égéidinne. Comme chez d'autres troglobies, les localités du genre Beskovia se trouvent aussi bien à l'Ouest qu'à l'Est de l'Iskar et cela confirme encore une fois l'hypothèse que plusieurs groupes de troglobies se sont formés et ont colonisé le domaine sousterrain avant l'entaillage épigénétique de la Stara planina par la rivière d'Iskar.

Dans plusieurs grottes de la Bulgarie du Nord-Ouest on trouve (surtout en automne) des Catopinae sous-troglophiles, appartenant à 8 espèces des genres Choleva, Catops et Nargus.

\section{RESUMÉ}

La région kartique qui s'étend entre les rivières Timok et $V$ it présente environ 500 grottes et gouffres, dont plus de 300 sont l'objet de la présente étude. Dans cette région (appelée ici Western Stara Planina), nous avons déterminé 
63 espèces et sous-espèces de troglobies terrestres et 17 espèces et sous-espèces de troglobies aquatiques. Cette région, que nous avons divisée en deux (Région d'Ogosta et Région d'Iskar), possède la faune cavernicole la plus riche de Bulgarie.

Cette étude traite des particularités de la composition et de la distribution des différents groupes de troglobies terrestres et surtout de groupes tels que les Diplopoda, Isopoda, Coleoptera et autres. D'après l'évolution paléogéographique de la région, des conclusions sont tirées au sujet de l'âge de certains troglobies et des barrières délimitant leur répartition actuelle.

\section{BIBLIOGRAPHIE}

ATANASOV N. et STEFANOV A., 1951, Die Höhle "Seeva dupka". -Bull. Inst. Zool., I, Sofia, 234-275.

BEAUCOURNU J.-C., 1967, Contribution à la connaissance de la biologie d'Ixodes (Eschatocephalus) vespertilionis Koch 1844 et d'Ixodes (Pomerantzevella) simplex Neumann 1906 (Acarina, Ixodoidea), parasites des Chiroptères.-Ann. Spéléol., XXII, 543-580.

BERON P., 1972, Essai sur la faune cavernicole de Bulgarie. III. Résultats des recherches biospéologiques de 1966 à 1970.-Int. j. Speleol., 4, 285-349.

BERON P. 1976. Subdivision zoogéographique de la Stara planina occidentale (Bulgarie) d'après sa faune cavernicole terrestre.-Acta zool. bulgarica, 4, 30-37.

BERON P. et V. GUÉORGUIEV, 1967, Essai sur la faune cavernicole de Bulgarie. II. Résultats des recherches biospéologiques de 1961 à 1965.-Bull. Inst. Zool., XXIV, Sofia, 151-212.

BONCEV E., 1971, (Problèmes de la géotectonique bulgare).- Ed. "Tehnika", Sofia, 204 pp

BOTOSANEANU L., 197i, Observations sur la faune aquatique hypogée des Monts du Banat (Roumanie).-Trav. Inst. Spéol., X, 123-166.

DECU, V. et ST. NEGREA, 1969, Aperçu zoogéographique sur la faune cavernicole terrestre de Roumanie. - Acta zool. Cracoviensia, XVI, 20, 471-545.

DELTSHEV, C.H., 1972, A New Genus of Bulgarian Cave Spiders (Protoleptoneta bulgarica n.g., n.sp., Leptonetidae). - Int. J. Speol., 4, 275-283.

DELTSHEV, CH., 1973, Redescription of Centromerus bulgarianus (Drensky, 1931) and Centromerus lakatnikensis (Drensky, 1931), (Araneae, Linyphiidae). - Int. J. Speleol., 5, 117-126.

DRENSKY, P., 1931, Höhlen-Spinnen aus Bulgarien. Rev. Acad. Bulg. Sci., XLIX, Sofia, 1-50.

DUSBÁBEK, FR., 1972, The zone of bat Acarinia in Central Europe. Folia parasitola (Praha), 19, 139-154.

FAGE, L., 1913, Etude sur les Araignées cavernicoles. II. Revision des Leptonetidae. Arch. Zool. Exp. gen., L, 479.

FAGE, L., 1931, Biospeleologica. LV. Araneae Cinquième série, précédée d'une essai sur l'évolution souterraine et son déterminisme. Arch. Zool. Exp. gén., 71, 99-291.

FUHN, I. E. et CL. OLTEAN, 1970, Lista araneelor dîn R. S. Romania. Museul de St. naturii Bacau, St. si Com., 157-196.

GRUIA, M., 1969, Date asupra raspindirii colembolelor in pesterile Romaniei. Lucr. inst. de Speol. "Emil Racovita", VIII, 161-178.

GUÉORGUIEV, V., 1973, Sur le rôle du linéament kraichtidovardarien en tant que barrière biogéographique durant le Tertiaire. C.R. Acad. bulg. Sci., 26, 533-535.

GUÉORGUIEV, V., 1974, Idées nouvelles sur la phylogénie et la paléozoogéographie de la sousfamille Bathysciinae (Catopidae, Coleoptera). C. R. Acad. bulg. Sci., 27, 965-968.

GUÉORGUIEV, V. et P. BERON, 1962, Essai sur la faune cavernicole de Bulgarie. Ann. Spéléol., XVII, 285-441.

HADZI, J., 1973, Catalogus faunae Jugoslaviae. III/4. Opilionidea. Ljubljana, 22 pp. 
JANETCHEK, H., 1957, Das seltsamste Tier Tirols. Palpenläufer (Arachn., Palpigrada): Stellung, Verbreitung, Arten, Bibliographie. Festschr. 50 jähr. Best. Kufst. Mittelschule 1907-1957.

JEANNEL, R., 1926, Faune cavernicole de la France avec une étude des conditions d'existence dans le domaine souterrain. Ed. Lechevalier, Paris, 334 pp.

JEANNEL, R., 1928, Monographie des Trechinae. Morphologie comparée et distribution géographique d'un groupe de Coléoptères (troisième livraison). Les Trechịni cavernicoles. L'Abeille, 35, $808 \mathrm{pp}$.

KRATOCHVIL, J., 1958a, Die Höhlenweberknechte Bulgariens (Cyphophthalmi und Laniatores). Prace brnenske Zakl. CSAV. XXX (9) 375, 372-396.

KRATOCHVIL, J., 1958b, Höhlenweberknechte Bulgariens (Palpatores-Nemastomatidae). Prace brnén. Zakl. CSAV. XXX (12), 379, 523-576.

KRATOCHVIL, J. et FR. MILLER, 1938, Sur le problème des Araignées cavernicoles du genre Centromerus de la péninsule Balkanique. Bull. Inst. roy. Hist. natur. Sofia, XI, 107-113.

KUZJAKIN, A., 1950, Les Chauves-sburis. Moscou.

LANEYRIE, R., 1970, Catalogue des Trechitae cryptiques. Publ. du Laboratoire Sout. du C.N.R.S. à Moulis, 45 pp.

LEROY, Y., 1967, Gryllides et Gryllacrides cavernicoles. Ann. Spéléol., XXII 659-722.

LERUTH, R., 1939, La biologie du domaine souterrain et la faune cavernicole de la Belgique. Mém. Mus. roy. Hist. Nat. Belg., 87, 506.

SIMON, E., 1911, Aran. et Opil. Ill Série. Biospeologica XXIII. Arch. Zool. Exp. gén., 49, 177-206.

STRASSER, K., 1966, Uber Diplopoden Bulgariens. Ann. Zool., XXIII, I2, 325-385.

STRASSER, K., 1974, Uber Diplopoda-Chilognatha Griechenlands. Rev. suise Zool., 81, 219-300. STRINATI, P., 1966, Faune cavernicole de la Suisse, Ann. Spéléol., 21, 5-483.

STRASSER, K., 1975, Weiterer Beitrag zur Kenntnis der bulgarischen Diplopoden. Acta zool. bulgarica, 3, 70-77.

TABACARU, J., 1970, Sur la répartition des Diplopodes cavernicoles europêens. 421-445. Livre du centenaire "Em. G. Racovitza" Bucarest 1970.

VANDEL, A., 1965, Les Isopodes terrestres et cavernicoles de la Bulgarie-Ann. Spéléol., XX, 243-270.

VANDEL, A., 1967, Les Isopodes terrestres et cavernicoles de la Bulgarie (second partie). Ann. Spéléol. XXII, 333-367.

VANDEL, A., 1968, Les Premiers Isopodes Terrestres et Cavernicoles découverets dans l'Archipel Nippon. Bull. Nat. Sci. Mus. Tokyo, 11 (4), p. 351-362.

VANDEL, A., 1969, Les Isopodes Terrestres et Cavernicoles de l'Archipel Nippon (Second Mémoire). Bull. Nat. Sci. Mus. Tokyo 13 (3), 373-383.

WILlMANN, C., 1941, Die Acari der Höhlen der Balkanhalbinsel. Stud. geb. allgem. Karstfor. wiss. Höhlenk., Biol. Ser., 8, 1-80.

VER HOEFF, K., 1941, Höhlen-Diplopoden aus dem Trentino. Z. Karst-Höhlenk., 166-173. 